\title{
REVISTAS CIENTÍFICAS: DETERMINACIÓN DE NECESIDADES Y USOS
}

\author{
María J. Reyes Barragán, Vicente P. Guerrero Bote, Antonio Pulgarín \\ Guerrero, Fellpe Zaplco Alonso*
}

Resumen: Se analizan tanto cuantitativa como cualitativamente las necesidades de información de la comunidad científica de las áreas de ciencia y tecnología de la Universidad de Extremadura a través de las citas a revistas. Para ello se lleva a cabo una búsqueda retrospectiva en la base de datos del Science Citation Index. Se consideraron múltiples variables, como usos por departamentos, factor de impacto, citas a revistas, disponibilidad, localización, accesibilidad y cobertura. A estas variables le fueron aplicadas una serie de indicadores (simples y complejos), recurriendo a modelos de asociacionismo múltiple (y en concreto al algoritmo de Kohonen), para determinar el grado de solapamiento interdepartamental en el consumo real de información.

Palabras clave: revistas científicas, citas, factor de impacto, redes neuronales, bibliometría, bibliotecas universitarias, Universidad de Extremadura.

\begin{abstract}
The information requirements of the scientific community of the areas of science and technology in the University of Extremadura were analysed quantitatively and qualitatively through journal citations. For this purpose, a retrospective search was made in the Science Citation Index database, considering multiple variables such as use by department, impact factor, journal citations, availability, localization, accessibility, and coverage. A set of (simple and complex) indicators was applied to these variables, using multiple association models (in particular, Kohonen's algorithm) to determine the degree of interdepartmental overlap in the real use of information.
\end{abstract}

Key words: scientific journals, citations, impact factor, neural networks, bibliometrics, university libraries, University of Extremadura.

\section{Introducción}

El presente trabajo es la aportación a un proceso llevado a cabo por la Universidad de Extremadura (UEX) que tiene como meta la optimización de todo el complejo engranaje del sistema bibliotecario propio de la Universidad.

La justificación de esta reorganización habría que buscarla tanto en factores intrínsecos, que afectan a la propia Universidad, como extrínsecos a ella, que afectarían al mundo de las bibliotecas, archivos y documentación, y en general al de la gestión documental. Entre los primeros cabría citar la evolución de la propia institución universitaria a la que los Servicios de Archivos Bibliotecas y Documentación deben

* Facultad de Biblioteconomía y Documentación de la Universidad de Extremadura. Badajoz. Correo-e: mjreyes@alcazaba.unex.es.

Recibido: Primera versión: 27-3-2000. Segunda versión: 2-11-2000. 
atender y con cuya propia dinámica de crecimiento y especialización habrán de sintonizar necesariamente. Entre los segundos, la globalización del mercado de la información hace que los sistemas de búsqueda y recuperación hayan de actualizarse continuamente alcanzando pronto unos niveles altos de obsolescencia. También la aparición de las nuevas tecnologías hace que los cambios respecto a la producción, transferencia, difusión y consumo de la información no sean ya de un rango cuantitativo sino que habrán de ser considerados como cualitativos.

Conocer y evaluar la realidad actual de los servicios de Archivos, Bibliotecas y Documentación de la UEX; sus éxitos, debilidades y por fin las posibilidades de un sistema que lleva en funcionamiento más de 25 años es el objetivo. De este conocimiento y análisis habrán de extraerse las conclusiones que nos permitirán, una vez conocidas las necesidades objetivas y subjetivas de todos los usuarios del sistema, trazar una serie de líneas maestras con las que podremos transformarlo haciéndolo más operativo, más flexible y, en definitiva, más rentable.

Para abordar con ciertas garantías de éxito toda esta compleja reestructuración, han de efectuarse determinados estudios que tienen que ver con los fondos con que cuenta actualmente la universidad, y que dada la estructura descentralizada de la que se parte se hallan dispersos por todos los centros que la conforman.

Por todo ello nos hacemos las siguientes preguntas: ¿proporciona la institución, las herramientas adecuadas a las necesidades reales de la comunidad científica? y ¿esta provisión se lleva acabo en las mejores condiciones? Al intentar responder a estas preguntas se hizo necesario plantearnos como objetivos conocer las necesidades de información de la comunidad científica de las áreas de ciencia y tecnología, a través de las revistas especializadas, su disponibilidad, accesibilidad y ubicación, por considerarse, hoy por hoy, las herramientas básicas con que cuentan los científicos como medio inmediato de transmisión de conocimientos y generador de otros nuevos que a su vez volverán a ser transmitidos.

\section{Análisis bibliométrico aplicado a la evaluación de revistas científicas}

Evaluar es medir, y medir es comparar. Como respuesta a las dos cuestiones fundamentales planteadas se habrá de comparar lo disponible y lo idóneo, para establecer los mecanismos y filtros necesarios para que los límites de ambos tiendan a unificarse. Vivimos en una época que ha sido definida acertadamente por los economistas como la de las expectativas ilimitadas; en la que los requisitos tienden a ser infinitos si se les deja evolucionar sin un acotamiento adecuado. Sin embargo, los medios son finitos, y vienen determinados por la capacidad de inversión. Esto puede aplicarse tanto a factores materiales como humanos que inciden en todo el proceso de información y gestión documental.

El puro ejercicio de la evaluación material de revistas científicas ha sido abordado desde dos puntos de vista y, como apunta Broude (1), por dos grupos de profesionales. Su concepción y ejecución tendentes a un mismo fin ofrece ciertas diferencias basadas predominantemente en la incidencia de factores «subjetivos», en relación con los criterios «objetivos». De cualquier forma es evidente que, sea cual fuere el camino emprendido, el mejor método será aquél que sirva mejor a las necesidades del centro concreto sobre el que se esté aplicando. 
Una relación exhaustiva de los criterios utilizados en clasificación para evaluación de publicaciones periódicas puede encontrarse en Lancaster $(2,3)$, Hawthorm (4) y Rashid (5). Algunos de estos criterios con alusión expresa del carácter objetivo/subjetivo se muestran en la tabla I.

Tabla I

Criterios de clasificación usados en la evaluación de publicaciones científicas

\begin{tabular}{|l|c|c|}
\hline \multicolumn{1}{|c|}{ Criterios de clasificación } & Objetivos & Subjetivos \\
\hline Utilización & & $*$ \\
Opinión usuarios/profesionales & $*$ & $*$ \\
Citas & $*$ & \\
Factor de impacto & $*$ & \\
Coste/eficacia & $*$ & \\
Accesibilidad/disponibilidad & $*$ & $*$ \\
Indización en bases de datos & $*$ & $*$ \\
Interés corporativo & $*$ & $*$ \\
Idioma & $*$ deputación del editor & \\
\hline
\end{tabular}

Podemos tomar como ejemplo o paradigma del primer modo (pesan aquí los factores subjetivos) el efectuado por Vals Pasola (6), en su estudio sobre cancelación de títulos en una biblioteca universitaria, y en el que la evaluación se lleva a cabo teniendo en cuenta el peso relativo de unas puntuaciones sobre uso, opinión, coste, pertinencia y oportunidad etc. Este estudio se sustancia en encuestas, en las que participaron profesores, usuarios y profesionales de las bibliotecas, y donde es evidente que influyen factores subjetivos en esa evaluación. Cabría citar otros estudios que se han desarrollado dentro de la misma línea y en otros países como los de González (7), y que son desarrollados por profesionales del sector.

Otros estudios, sin embargo, tienen preferente y exclusivamente en cuenta criterios objetivos, como el realizado por E. Jiménez-Contreras (8), en el que propone como método de evaluación contar casi exclusivamente con las citas, que «son en definitiva una expresión del uso de los fondos». En este sentido su opción concuerda con la de Altuna y Lancaster (9). Es notorio que los especialistas en esta materia defienden la prevalencia de los criterios objetivos sobre los subjetivos, y entendemos como más interesante esta opción; aún sabiendo el sesgo que establecen las bases de datos utilizadas, pero que son, sin embargo, las únicas herramientas de que se dispone para cuantificar.

La metodología que propone Jiménez-Contreras para determinar la colección básica de publicaciones periódicas en hemerotecas científicas, se basa en el recuento de las revistas utilizadas por un grupo de científicos para publicar, pertenecientes a un área determinada, así como en el recuento de citas a revistas científicas, contenidas en dichos trabajos, apoyándose en la ley de Bradford, modificada por Brookes y Ferreiro. Este autor establece asimismo el tiempo estimado necesario para este tipo de estudios, y finalmente una rutina de obtención y agrupación de datos, llegando a la con- 
clusión que este tipo de estudios ha de completarse con otros que lleven en sí cierto grado de «opinión» si bien el grueso fundamental de la evaluación gravita en la metodología descrita.

\section{Metodología}

La metodología sigue, esencialmente, la propuesta por Jiménez-Contreras, basada en el recuento de citas.

\subsection{Fuentes de datos}

Para poder llevar a cabo el recuento de citas a revistas científicas, en las áreas de ciencia y tecnología, se recurrió a la base de datos del SCI (Science Citations Index). Se establecieron las búsquedas por el campo «address» (dirección de los autores pertenecientes a la UEX). Se obtuvo así la producción de la UEX en estos campos, en un período de tiempo de dos años (1996/1997), ya que se consideró este período de tiempo suficiente para determinar las necesidades de información de los científicos. El formato de registro recuperado del SCI incluía los siguientes campos: autor, título, fuente de publicación, lengua, tipo de documento, registros relacionados, número de referencias y referencias citadas.

\subsection{Análisis de los datos}

Se establecieron una serie de criterios para la ordenación de los datos obtenidos con el fin de abordar los objetivos planteados, esto es, conocer el consumo de información, disponibilidad, accesibilidad y determinación de la colección básica.

\subsubsection{Criterios de ordenación de los datos}

En esta etapa se procedió a una estructuración de los registros bibliográficos recuperados de la base de datos respecto a una serie de criterios: $a$ ) los obtenidos de los propios campos de los registros; $b$ ) los obtenidos a partir de fuentes externas. Esta clasificación se hizo en base a:

\subsubsection{Citas a revistas}

A partir de las referencias bibliográficas obtenidas de los registros, se extrajo el número de citas que recibía cada título de revista del campo «cited references», se intentó detectar el consumo de información y constituir el corpus de revistas más citadas en las áreas de ciencia y tecnología de nuestra universidad, consideradas éstas como un criterio objetivo que mide el consumo de información real a través de las propias referencias. 


\subsubsection{Usos de revistas por departamentos}

Este criterio es mas complejo ya que puede estar sujeto a cierta controversia a la hora de evaluar las publicaciones periódicas, aunque resulta necesario para poder llegar a establecer la propuesta de la colección básica y subsanar de alguna manera la problemática que se da en torno a su ubicación, de forma que quede accesible para todos. No se trata ya de delimitar cuáles son las necesidades de información de un grupo de científicos, sino cómo determinar el consumo de información medido en usos reales por los departamentos a los que pertenecen los científicos. Por ello es imprescindible considerar dos cuestiones: ¿cómo se han establecido los usos de revistas en los departamentos? y ¿cuál es la organización científica de la institución objeto de estudio?

Respecto a la primera cuestión, hay que tener en cuenta las dificultades que entraña el cuantificar los usos de las colecciones, y en concreto de las publicaciones periódicas, tal y como aparecen recogidas en la bibliografía sobre el tema (10). Aquí hay que plantearse qué es un uso en una biblioteca: cuando una determinada revista se toma de la estantería y se hojea, o cuando se extrae y se lleva a la mesa.

El propósito sería detectar qué revistas se están utilizando y quiénes las utilizan; sirviendo lo primero para la política de adquisiciones y lo segundo para determinar su ubicación. Para ello se creó un fichero de autores de los trabajos en ciencia y tecnología de la universidad, encontrando dificultades para la identificación exhaustiva y unívoca de los autores productivos. Debido a la falta de normalización de los nombres en la base de datos del SCI, en especial de aquéllos que firman con dos apellidos, este problema se acentúa cuando éstos son muy comunes, y en caso de contener partículas, situación ésta provocada, en parte, por los propios autores que no siempre firman de igual manera, y al aplicar indistintamente las normas establecidas para autores españoles y anglosajones (11).

Una vez identificados los autores firmantes de cada trabajo, fueron asignados al departamento al que pertenecían. De igual modo, las referencias bibliográficas recogidas en cada trabajo también eran asignadas a cada departamento, aunque ponderadas (multiplicadas) por el número de firmantes de un trabajo pertenecientes a un departamento.

\subsubsection{Factor de impacto}

Las instituciones que promueven la investigación desarrollan habitualmente actividades de evaluación orientadas principalmente al incremento de su propia calidad investigadora; para ello se suele utilizar el JCR (Journal Citation Report), clasificación temática de las revistas científicas ordenadas en función de índice de impacto. Aunque este indicador ha sido objeto de críticas por parte de la comunidad científica, es utilizado como instrumento casi exclusivo en la medición de la calidad científica y está ya prácticamente establecida su admisión para tal fin a nivel mundial.

Se asignó su factor de impacto a todas las revistas citadas en la producción científica de la institución en los dos últimos años, para poder determinar el prestigio y visibilidad de las revistas usadas y establecer una comparación entre los distintos departamentos a partir de las publicaciones que presentaban un número de usos igual o superior a diez. En el mismo registro se han incluido el factor de impacto y el número de citas, para determinar si las revistas más citadas por los científicos de la institución son aquéllas que tienen un mayor factor de impacto. 


\subsection{Tratamiento informático}

Ordenados los datos, en base a los criterios anteriormente mencionados, se procedió a su tratamiento informático, donde se siguieron los siguientes pasos:

a) En primer lugar se diseño una serie de tablas (siguiendo el modelo relacional) cuya estructura y campos se muestran en la tabla II.

Tabla II

Estructura de datos de la base relacional diseñada para el proceso informático

\begin{tabular}{|c|c|c|c|c|}
\hline Tabla & Atributo & Tipo & Tamaño & Claves \\
\hline$L d p$ & $\begin{array}{l}\text { Clave_dep } \\
\text { Numérico } \\
\text { Abreviado }\end{array}$ & $\begin{array}{l}\text { Numérico } \\
\text { Carácter } \\
\text { Carácter }\end{array}$ & $\begin{array}{l}2 \\
50 \\
15\end{array}$ & [Clave Primaria] \\
\hline Laut & $\begin{array}{l}\text { Clave_aut } \\
\text { Clave_dep } \\
\text { Nombre }\end{array}$ & $\begin{array}{l}\text { Numérico } \\
\text { Numérico } \\
\text { Carácter }\end{array}$ & $\begin{array}{l}3 \\
2 \\
30\end{array}$ & $\begin{array}{l}\text { [Clave Primaria] } \\
\text { [Clave Secund.] }\end{array}$ \\
\hline Trabajos & $\begin{array}{l}\text { Clave_art } \\
\text { Título }\end{array}$ & $\begin{array}{l}\text { Numérico } \\
\text { Carácter }\end{array}$ & $\begin{array}{l}3 \\
50\end{array}$ & [Clave Primaria] \\
\hline Lrevist & $\begin{array}{l}\text { Clave_rev } \\
\text { Citas } \\
\text { Nombre } \\
\text { F. impacto } \\
\text { Cobertura }\end{array}$ & $\begin{array}{l}\text { Numérico } \\
\text { Numérico } \\
\text { Carácter } \\
\text { Numérico } \\
\text { Numérico }\end{array}$ & $\begin{array}{l}4 \\
4 \\
40 \\
20 \\
2\end{array}$ & [Clave Primaria] \\
\hline
\end{tabular}

b) En segundo lugar se desarrollaron varios programas informáticos para extraer estos datos de los registros bibliográficos obtenidos del SCI. Algunos campos se tuvieron que completar manualmente, como es el caso del departamento al que pertenece cada autor, el factor de impacto de cada publicación periódica y la disponibilidad de las mismas en las bibliotecas de la Universidad de Extremadura.

c) De forma paralela se realizaron los programas encargados de generar los vectores, que posteriormente fueron procesados mediante el algoritmo de Kohonen (12).

\subsection{Ley de Bradford. Determinación del núcleo}

La determinación del núcleo se realizó sobre la distribución de citas a revistas. Seguimos en este trabajo el método propuesto por Ferreiro (15), modificado de Brookes (16), siendo la expresión matemática utilizada:

$$
R(n) \cdot \log \frac{n}{x_{1}}+y_{1}
$$




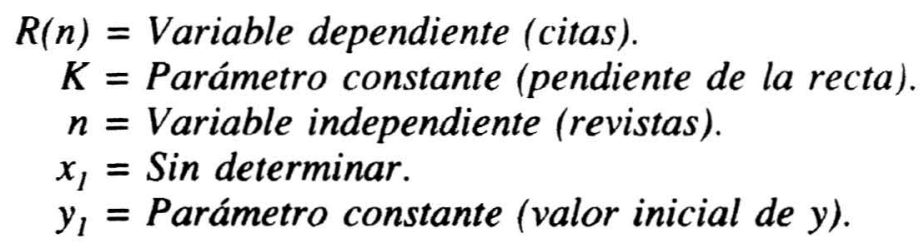

No obstante, queremos hacer tres consideraciones que no por obvias o conocidas dejan de ser importantes: la fracción recta a la que «aspira» toda la distribución será aquélla que se encuentre dentro del valor que se quiera asumir como idéntico entre los datos observados y los calculados. Nosotros, para el presente trabajo, y teniendo en cuenta la recta obtenida, proponemos «dentro de la recta» una diferencia menor o igual al $1 \%$ entre los valores observados y los calculados con un coeficiente de correlación del 0,999 en la distribución de citas a revistas.

\subsection{Disponibilidad en biblioteca}

Este trabajo quedaría inconcluso si sólo analizáramos una serie de parámetros o variables en función de las revistas científicas, como los utilizados hasta ahora: usos por departamentos, citas a revistas científicas, factor de impacto, etc.; sino que el objetivo es determinar hasta qué punto las revistas científicas más usadas y citadas guardan relación con la disponibilidad que tienen los usuarios de éstas, a través del análisis del nivel de cobertura en las bibliotecas de los distintos centros, en las áreas de ciencia y tecnología. Debemos aclarar que el criterio seguido para determinar esta cobertura ha sido fijado en base a: suscripciones vivas a revistas científicas; suscripciones pagadas por la propia institución, excluyéndose aquéllas de carácter personal; revistas científicas que han sido utilizadas en la clasificación departamental con una frecuencia superior a 10 usos y revistas científicas que en la clasificación por citas presentan una frecuencia superior a 5 citas.

Asimismo no se ha tenido en cuenta el nivel de duplicidad existente, que siempre ha sido éste un criterio muy tenido en consideración a la hora de efectuar cancelaciones, y que quedaría justificado con un alto índice de uso en departamentos y centros, aunque somos conscientes de las ventajas que ofrecen las tecnologías de la información, acceso a redes, ediciones electrónicas, etc., que su uso cada vez más generalizado está dando lugar a nuevas formas de comunicación científica $(17,18)$.

\subsection{Clasificación de las revistas en base a los usos recibidos}

Demanda y accesibilidad van estrechamente ligadas, ya que la idea intuitiva de que lo que más se usa suele estar más a la mano, es algo que se cumple en la vida diaria.

En la institución que nos ocupa, y por su propia estructura, las revistas en formato papel están depositadas en departamentos (la mayoría) y bibliotecas de centro, dándose la circunstancia de estar dispersos los títulos disponibles, y con diversos grados de accesibilidad, que hace que ésta no sea paritaria para docentes, estudiantes, investigadores, etc. 
Determinados los usos de revistas por los distintos departamentos, según las citas recibidas ponderadas por el número de autores de cada departamento, se clasifican los títulos en base a dichos usos. De este modo, tendremos una especie de foto o mapa de cómo ven los científicos de la Universidad de Extremadura las distintas revistas.

Para ello vamos a utilizar un algoritmo que se puede encuadrar dentro de las ANN (Artificial Neural Networks o Redes Neuronales Artificiales) (19). El modelo de red neuronal que vamos a aplicar se debe a Kohonen, y tiene la particularidad de clasificar las revistas (entradas) sobre una rejilla (hexagonal). De este modo, en cada neurona o nodo de la rejilla se forma un cluster de revistas similares. Además, clasifica los clusters topológicamente sobre la rejilla, es decir, que las revistas clasificadas en clusters vecinos tienen un mayor parecido que las clasificadas en clusters alejados. Esto nos permite tener una vision topológica de las revistas, clasificadas según los usos realizados por los departamentos, que nos puede servir incluso para determinar la localización de cada una de ellas.

Para utilizar este modelo, igual que para utilizar cualquier otro algoritmo de clustering o de reducción de la dimensión, se hace necesario representar las revistas (entradas) vectorialmente. En nuestro caso, como ya hemos indicado, se trata de representarlas en función de los usos que reciben de los departamentos; para ello se utilizan vectores de ventiún componentes, que ponderan los usos de los veintiún departamentos respectivamente. En esa ponderación se utilizó un esquema de pesos similar al IDF (Inverse Document Frecuency) utilizado habitualmente para la indización automática de los documentos en el modelo del espacio vectorial $(13,14)$ :

$$
a_{i j}=d_{i j} \cdot \log \frac{P}{P_{j}}
$$

donde:

$a_{i j}=$ Peso asignado al uso de la revista i-ésima por el departamento j-ésimo (es decir, el peso asignado al uso de una revista en un departamento).

$d_{i j}=$ Número de usos de la revista i-ésima por el departamento j-ésimo (número de usos de una revista en un departamento).

$p_{j}=$ Número de revistas utilizadas por el departamento j-ésimo.

$P=$ Número total de revistas.

La ventaja de este modelo frente a otros métodos de reducción de la dimensión radica en la posibilidad de clasificar un gran número de revistas y localizar dónde estaría ubicado hipotéticamente el departamento.

\section{Resultados}

La búsqueda en el SCI de la producción científica de la Universidad de Extremadura en 1996-1997 arroja un total de 375 trabajos, de ellos el $88 \%$ corresponde a artículos científicos, que suponen el $47 \%$ del total de artículos publicados en esos años por investigadores de la UEX en los campos de ciencia y tecnología (tomando como fuente las memorias de investigación de la UEX). 


\subsection{Usos por departamentos}

En la tabla III se recoge la distribución de todos los departamentos analizados relacionados con ciencia y tecnología (21 departamentos) y expresados mediante un código (Anexo I), donde se han aplicado una serie de indicadores, para hacer un análisis cuantitativo y cualitativo y poder determinar el consumo real de información.

En cuanto al número de títulos de revistas citadas se observa una gran diferencia entre unos y otros departamentos, determinado en gran medida por el hecho de que sean departamentos consolidados en el tiempo, que a su vez son los más productivos. Tampoco hay que olvidar el sesgo que marca el número de referencias utilizadas en los trabajos.

Paralelamente se estudia el número de revistas cuya frecuencia de uso es superior a 10, acusándose la misma polidispersión interdepartamental, pudiendo establecerse una relación casi lineal entre el número absoluto de revistas y aquéllas cuyos usos son iguales o superiores a 10.

Respecto a la media de usos de las revistas citadas por departamentos y de aquéllas cuyos usos superan la frecuencia 10, habremos de notar que éstos corresponden en mayor medida a ciencias puras, concretamente a las áreas de física y química.

En cuanto al factor de impacto, tanto del total de revistas citadas como de aquéllas con frecuencia superior a 10 usos, es indicativo de la calidad por departamentos, pero no es recomendable para establecer comparaciones interdepartamentales, ya que el JCR establece impactos por áreas temáticas, que si bien hemos intentado asimilarlos a los departamentos no tienen por qué ser coincidentes en todo ni en todos los casos. Para hacer esta relación hemos introducido todas las áreas del JCR afines al departamento en cuestión cogiendo como indicador de cada clase el percentil 50, y se ha aplicado el promedio de éstas al departamento correspondiente, hallándose que todos los departamentos superan el promedio de la mediana en cuanto a F.I., destacando las áreas biomédicas que casi cuadruplican el promedio, al ser éstas las que resultan ser puntas y de vanguardia en investigación.

La correlación que puede establecerse entre los usos mayores de 10 y el F.I. es casi inexistente, contra lo que a priori cabría esperar, dando por cierto que el número de usos debería ser función directa de la calidad. Esta distorsión podría venir dada por los valores extremos de la distribución, por la incorporación de revistas multidisciplinares con alto factor de impacto y áreas de conocimiento con poca productividad que, al introducir revistas muy específicas de su área con pocos usos y alto F.I., distorsionan la distribución.

Por último, hemos plasmado el porcentaje de revistas con usos superiores a 10 por departamentos, disponibles en bibliotecas de la institución, produciéndose igualmente una polidispersión.

\subsection{Citas a revistas}

Tras aplicar la Ley de Bradford al conjunto de revistas citadas se obtuvo que el núcleo estaba determinado por 30 revistas, que suponen el 1,22\% del total de revistas citadas, en donde se concentran el $27,32 \%$ de las citas a revistas. Para la determinación de éste se ha admitido una discrepancia superior al $1 \%$ entre el tramo recto y curvo y un coeficiente de correlación del 0,999 . 
Tabla III

Distribución de usos por departamentos en relación al total de revistas citadas

\begin{tabular}{|c|c|c|c|c|c|c|c|c|c|c|}
\hline $\begin{array}{l}\text { Cod. } \\
\text { Dep. }\end{array}$ & $\begin{array}{l}\text { Revis- } \\
\text { tas }\end{array}$ & $\begin{array}{c}\text { Revistas } \\
(\text { usos } \geq 10)\end{array}$ & $\%$ & $\begin{array}{l}\text { Promedio } \\
\text { de usos }\end{array}$ & $\begin{array}{c}\text { Promedio } \\
\text { usos (rev. usos } \\
\geq 10)\end{array}$ & $\begin{array}{c}\text { Promedio } \\
\text { FI. }\end{array}$ & $\begin{array}{l}\text { Promedio F.I. } \\
\text { (rev. usos } \\
\geq 10)\end{array}$ & $\begin{array}{l}\text { Promedio de } \\
\text { medianas } \\
\text { de FI. }\end{array}$ & $\begin{array}{l}\text { Correlación- } \\
\text { F.I. (usos } \\
\geq 10)\end{array}$ & $\begin{array}{l}\text { \% disponible } \\
\text { (usos } \geq 10 \text { ) }\end{array}$ \\
\hline 1 & 126 & 21 & 16,67 & 10,28 & 47,33 & 1,42 & 2,23 & 1.065 & 0,1569 & 23,81 \\
\hline 2 & 65 & 1 & 1,54 & 1,86 & 16,00 & 3,34 & 3,32 & - & - & 100,00 \\
\hline 3 & 268 & 26 & 9,70 & 6,12 & 40,27 & 2,81 & 7,58 & 1,798 & $-0,0835$ & 42,31 \\
\hline 4 & 47 & 1 & 2,13 & 1,70 & 13,00 & 1,54 & 1,86 & - & - & 100,00 \\
\hline 5 & 251 & 26 & 10,36 & 5,64 & 29,35 & 2,31 & 6,50 & 1,098 & 0,2452 & 30,77 \\
\hline 6 & 99 & 1 & 1,01 & 1,66 & 13,00 & 2,56 & 1,67 & - & - & 100,00 \\
\hline 7 & 80 & 19 & 23,75 & 8,13 & 24,84 & 5,06 & 10,78 & 1,514 & $-0,1615$ & 68,42 \\
\hline 8 & 354 & 37 & 10,45 & 4,54 & 19,51 & 1,49 & 1,99 & 1,106 & 0,0048 & 21,62 \\
\hline 9 & 154 & 17 & 11,04 & 5,28 & 23,29 & 1,78 & 1,47 & 0,957 & $-0,5517$ & 23,53 \\
\hline 10 & 162 & 28 & 17,28 & 11,22 & 47,54 & 2,53 & 3,85 & 1,110 & $-0,1132$ & 35,71 \\
\hline 11 & 563 & 58 & 10,30 & 5,60 & 32,78 & 0,65 & 1,78 & 0,938 & $-0,0157$ & 18,97 \\
\hline 12 & 358 & 69 & 19,27 & 7,54 & 25,93 & 2,61 & 4,69 & 1,335 & 0,0725 & 20,29 \\
\hline 13 & 141 & 29 & 20,57 & 9,90 & 35,24 & 1,05 & 1,60 & 0,545 & $-0,0822$ & 27,59 \\
\hline 14 & 168 & 26 & 15,48 & 5,70 & 20,12 & 1,42 & 1,92 & 0,670 & 0,1849 & 46,15 \\
\hline 15 & 105 & 1 & 0,95 & 1,63 & 10,00 & 3,49 & 7,45 & 0,890 & - & 100,00 \\
\hline 16 & 207 & 28 & 13,53 & 5,99 & 26,25 & 2,72 & 5,06 & 1,424 & 0,1314 & 32,14 \\
\hline 17 & 370 & 42 & 11,35 & 5,77 & 28,83 & 0,96 & 1,88 & 0,799 & 0,0919 & 21,43 \\
\hline 18 & 66 & 0 & 0,00 & 1,56 & 0,00 & 0,49 & 0,00 & 0,632 & - & 0,00 \\
\hline 19 & 217 & 13 & 5,99 & 3,49 & 23,00 & 0,96 & 1,50 & 0,344 & $-0,1337$ & 0,00 \\
\hline 20 & 56 & 0 & 0,00 & 2,41 & 0,00 & 1,20 & 0,00 & 0,402 & - & 0,00 \\
\hline 21 & 84 & 0 & 0,00 & 1,87 & 0,00 & 3,58 & 0,00 & 0,867 & - & 0,00 \\
\hline
\end{tabular}


La determinación de la $1 .^{\mathrm{a}}$ zona engloba 151 revistas que suponen un $6,12 \%$ del total y 2.500 citas que suponen un $27,14 \%$ del total, de modo que el $54,46 \%$ de las citas lo soportan 181 revistas que suponen un $7,34 \%$ del total de revistas citadas en las áreas de ciencia y tecnología de la UEX.

Tal como se observa en la tabla IV, se ha aplicado una serie de indicadores a la distribución de citas a revistas, como la correlación entre citas y usos. Los valores de citas y usos obtenidos, tanto en toda la distribución como en el núcleo, 1." zona y núcleo más primera zona son de una dependencia muy fuerte. En cuanto a la correlación entre citas y factor de impacto, los valores obtenidos están próximos al valor nulo en el núcleo y $1 .^{a}$ zona, lo que indica la inexistencia de una asociación lineal entre los valores considerados entre las variables de la distribución. En la secuencia general y en el núcleo más $1 .^{a}$ zona sí se observa una dependencia muy baja en el coeficiente de correlación.

Tabla IV

Distribución de citas a revistas. Indicadores

\begin{tabular}{|l|r|r|r|c|}
\hline & General & Núcleo & \multicolumn{1}{|c|}{ l. $^{a}$ zona } & Núcleo+1.a zona \\
\hline Correlación entre citas y usos & 0,95 & 0,88 & 0,81 & 0,93 \\
Correlación entre citas y F.I. & 0,15 & $-0,04$ & 0,01 & 0,11 \\
Promedio de citas & 3,73 & 83,87 & 16,56 & 27,71 \\
Promedio de usos & 9,16 & 192,97 & 42,25 & 67,23 \\
Disponibilidad en biblioteca & 90,00 & 17,00 & 38,00 & 55,00 \\
Promedio F.I. & 2,66 & 6,50 & 3,75 & 4,26 \\
\hline
\end{tabular}

En lo referente a disponibilidad en bibliotecas en la distribución de citas a revistas, encontramos disponibles con carácter institucional 90 revistas (sólo se han tenido en cuenta aquéllas superiores a 10 usos y 5 citas, siendo éstas 270 revistas), que suponen un 33\% de las revistas citadas con estas características y un 3,6\% en relación al total. De éstas, $17(57 \%)$ están disponibles en el núcleo de un total de 30; en la 1. ${ }^{a}$ zona se detectaron $38(25 \%)$ de un total de 151 títulos, quedando ambos bastante al descubierto.

En la tabla $\mathrm{V}$ se han recogido para cada uno de los departamentos el total de revistas citadas, las revistas con más de nueve usos, la disponibilidad en bibliotecas, la cobertura y el incremento que proporcionaría el núcleo y la primera zona de las revistas más citadas por departamento. En general, se han observado valores muy dispersos, tanto en la disponibilidad en bibliotecas de revistas con más de nueve usos, como en la cobertura que ofrecería el conjunto de revistas más citadas en núcleo y primera zona por departamentos.

No obstante, si aceptamos como «colección básica de revistas» la determinada por el núcleo y 1. a zona, la cobertura que ésta ofrecería a los departamentos, tal y como aparece en la figura 1, sería siempre superior a los valores obtenidos para cada uno de los departamentos, con una frecuencia igual o superior a 10 usos, y que presentarían como característica general que son revistas con más de 10 usos e igual o superior a 9 citas. Los incrementos que se producirían en los departamentos por la cobertura del núcleo más 1. a zona oscilarían ente el $100 \%$ (Fisiología) y el $3.600 \%$ 
Tabla V

Cobertura del núcleo y primera zona en relación a los departamentos

\begin{tabular}{|c|c|c|c|c|c|c|c|c|c|}
\hline $\begin{array}{l}\text { Cod. } \\
\text { Dep. }\end{array}$ & $\begin{array}{l}N^{o} \\
\text { rev. }\end{array}$ & $\begin{array}{c}N^{\circ} \text { rev. } \\
(\text { usos } \geq 10)\end{array}$ & $\%$ & $\begin{array}{l}\text { Dispo- } \\
\text { nibles }\end{array}$ & $\begin{array}{c}\text { No } \\
\text { dis- } \\
\text { ponibles }\end{array}$ & Resto & $\begin{array}{c}N .^{o} \\
\text { revistas } \\
N+1 .^{a} Z\end{array}$ & $\%$ & $\begin{array}{c}\text { \% incremento } \\
\text { en cobertura } \\
N+1 .^{a} Z .\end{array}$ \\
\hline 1 & 126 & 21 & 16,67 & 5 & 16 & 98 & 28 & 22,22 & 133,33 \\
\hline 2 & 65 & 1 & 1,54 & 1 & 0 & 36 & 29 & 44,62 & $2.900,00$ \\
\hline 3 & 268 & 26 & 9,70 & 11 & 15 & 211 & 57 & 21,27 & 219,23 \\
\hline 4 & 47 & 1 & 2,13 & 1 & 0 & 42 & 5 & 10,64 & 500,00 \\
\hline 5 & 251 & 26 & 10,36 & 8 & 18 & 214 & 37 & 14,74 & 142,31 \\
\hline 6 & 99 & 1 & 1,01 & 1 & 0 & 79 & 20 & 20,20 & $2.000,00$ \\
\hline 7 & 80 & 19 & 23,75 & 13 & 6 & 47 & 33 & 41,25 & 173,68 \\
\hline 8 & 354 & 37 & 10,45 & 8 & 29 & 299 & 55 & 15,54 & 148,65 \\
\hline 9 & 154 & 17 & 11,04 & 4 & 13 & 124 & 30 & 19,48 & 176,47 \\
\hline 10 & 162 & 28 & 17,28 & 10 & 18 & 126 & 36 & 22,22 & 128,57 \\
\hline 11 & 563 & 58 & 10,30 & 11 & 47 & 510 & 53 & 9,41 & 91,38 \\
\hline 12 & 358 & 69 & 19,27 & 14 & 55 & 289 & 69 & 19,27 & 100,00 \\
\hline 13 & 141 & 29 & 20,57 & 8 & 21 & 119 & 22 & 15,60 & 75,86 \\
\hline 14 & 168 & 26 & 15,48 & 12 & 14 & 133 & 35 & 20,83 & 134,62 \\
\hline 15 & 105 & 1 & 0,95 & 1 & 0 & 69 & 36 & 34,29 & $3.600,00$ \\
\hline 16 & 207 & 28 & 13,53 & 9 & 19 & 173 & 34 & 16,43 & 121,43 \\
\hline 17 & 370 & 42 & 11,35 & 9 & 33 & 327 & 43 & 11,62 & 102,38 \\
\hline 18 & 66 & 0 & 0,00 & 0 & 0 & 63 & 3 & 4,55 & - \\
\hline 19 & 217 & 13 & 5,99 & 0 & 13 & 180 & 37 & 17,05 & 133,33 \\
\hline 20 & 56 & 0 & 0,00 & 0 & 0 & 45 & 11 & 19,64 & - \\
\hline 21 & 84 & 0 & 0,00 & 0 & 0 & 63 & 21 & 25,00 & - \\
\hline
\end{tabular}




\section{Figura 1}

Cobertura del núcleo y primera zona por departamentos

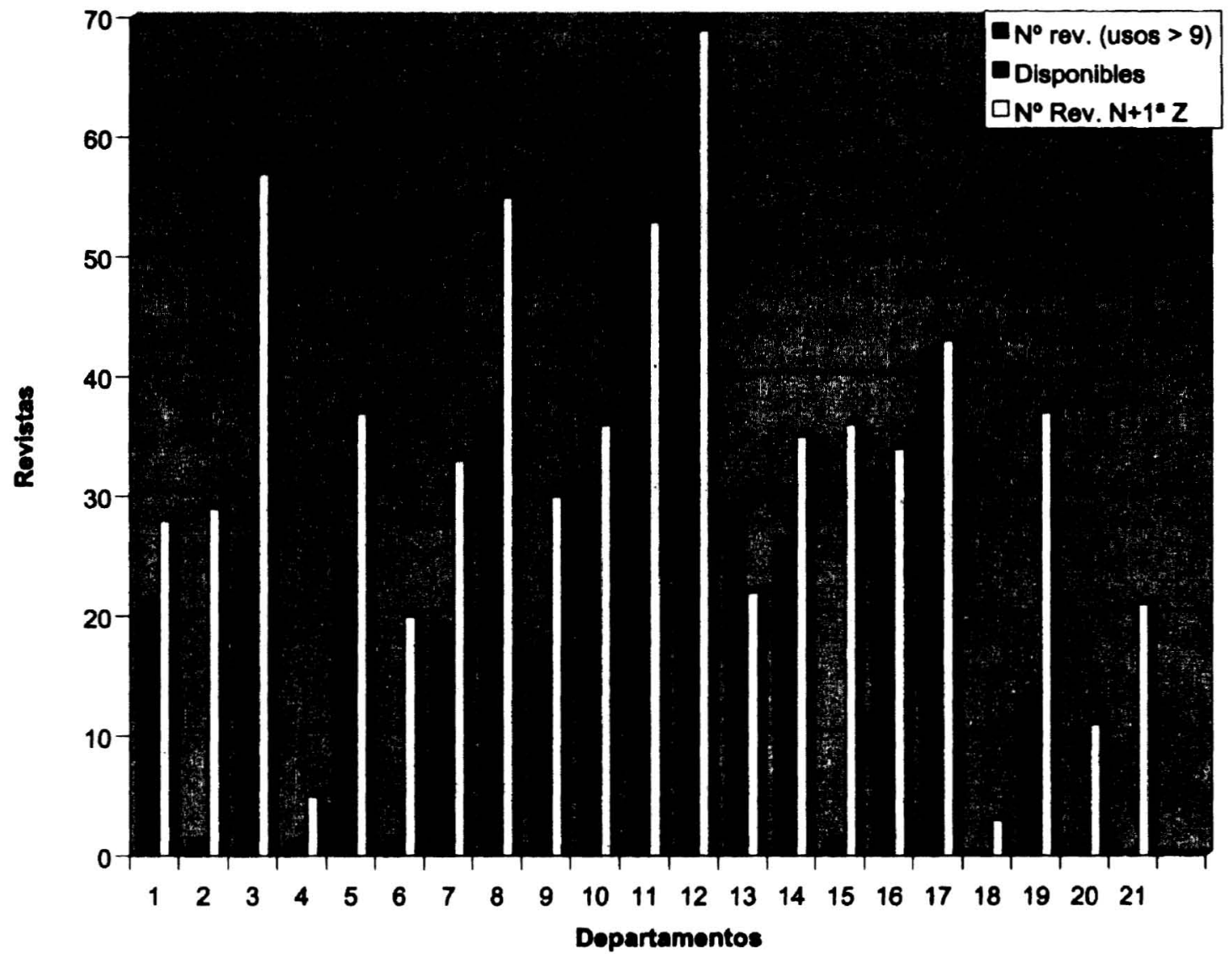

(Patología y Clínicas Humanas). En éstos se incluirían casi todas las revistas con más de 10 usos, se abarcarían otras con carácter multidisciplinar que quedaron excluidas en algunos departamentos y otras con menor número de usos. En general, esta situación se da para casi todos los departamentos. Estos incrementos serían siempre revistas compartidas por varios departamentos. Solamente en los departamentos de Ingenierías Químicas y Energéticas y Fisiología descenderían los valores de cobertura, porque aunque son revistas con más de 10 usos, no superan las 9 citas, que es el límite de la primera zona de revistas.

\subsection{Clasificación de las revistas en base a los usos recibidos}

Los usos de revistas científicas por departamentos nos permitirán determinar el nivel óptimo de localización y accesibilidad, y para ello hemos recurrido a los modelos de asociacionismo múltiple y en concreto al algoritmo de Kohonen, capaz de determinar el comportamiento de los departamentos en materia de usos a revistas y así organizar topológicamente las revistas (entradas).

La red utilizada era de 20 × 30 nodos y de una vecindad hexagonal, lo que quie- 
re decir que da lugar a una rejilla hexagonal de esas dimensiones como se puede observar en la figura 2. Los puntos negros corresponden a las neuronas, es decir, a los nodos de la rejilla, y en ellos han sido clasificadas las revistas. Las gradaciones de cofor indican las distancias existentes entre las neuronas, o lo que es lo mismo, la exis

Figura 2

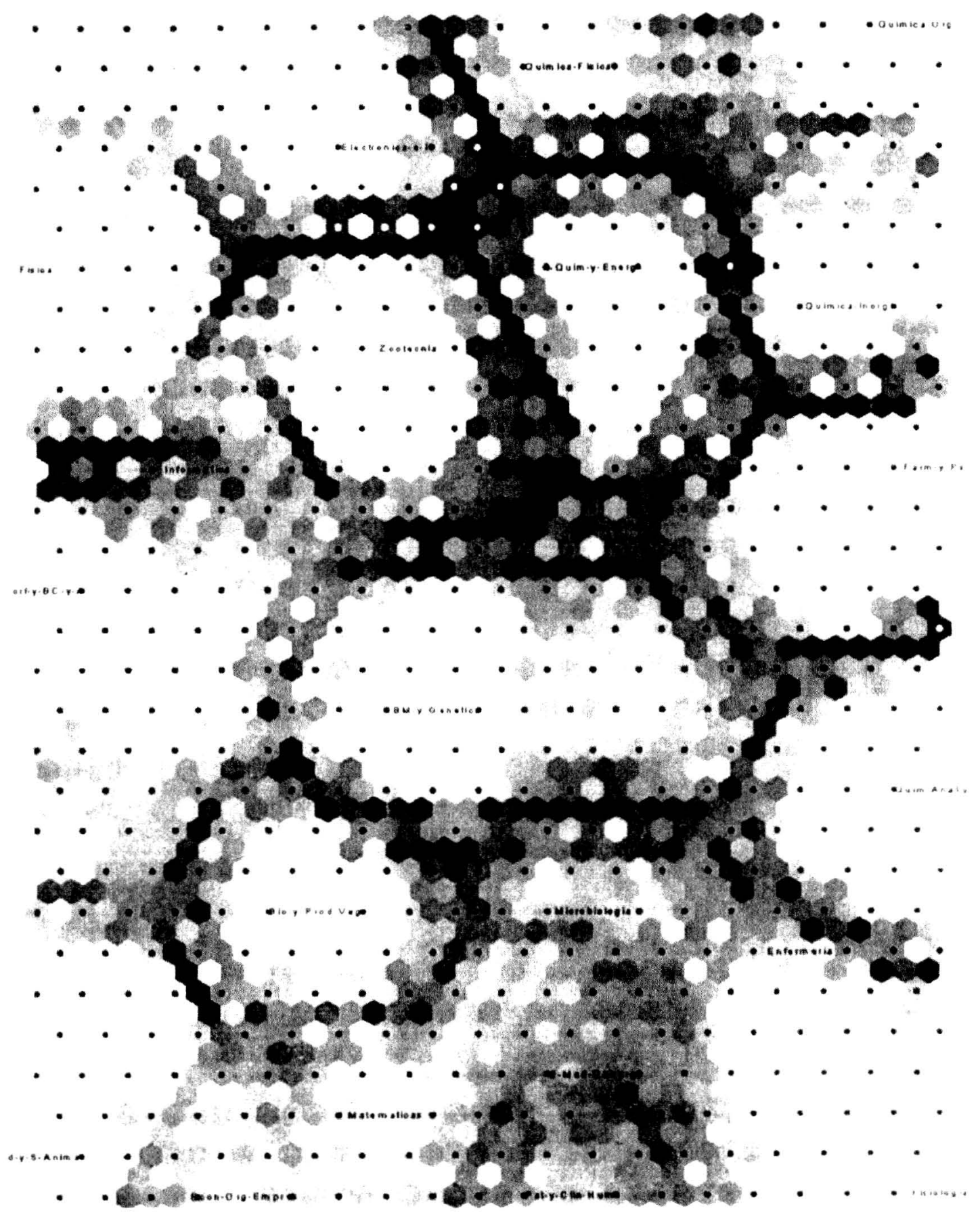


tente entre los grupos de revistas a que dan lugar. De este modo, las neuronas se pueden ver como poblaciones donde se agrupan las revistas, mientras que las gradaciones de color, igual que en algunos mapas, pueden indicar la presencia de montañas entre las poblaciones, lo que se traduce en aislamiento entre ellas y diferencias entre sus habitantes. En nuestro caso, las revistas con un patrón de usos similar se han clasificado en la misma neurona, y los nodos situados dentro del mismo valle contienen revistas con patrones bastante similares.

Un primer dato que podemos obtener de esta representación es que los valles están asociados a un departamento, y en la mayoría de los casos todos los departamentos tienen aparejado su valle. Esto quiere decir que al clasificar las revistas surgen valles donde se agrupan las revistas que usa principalmente un departamento. Algunos de los mismos están más definidos que otros, indicando que existe un grupo definido de revistas utilizado mayoritariamente por el departamento correspondiente. Otras zonas son más grises, como mesetas, que indican una zona de solapamiento entre departamentos. Por último, existen zonas oscuras (montañas) que marcan el aislamiento entre valles, como aparece reflejado entre los clusters de Ingeniería Química y Energética, Química-Física, Ingeniería Electrónica y Zootecnia. En dichas zonas oscuras suele haber muy pocas revistas y no hay departamentos salvo raras excepciones. Dichas excepciones corresponden a departamentos cuya investigación no está muy consolidada y utiliza revistas asociadas a los departamentos circundantes, como es el caso de Informática.

Si observamos el caso del departamento de Bioquímica y Biología Molecular y Genética, las fronteras oscuras se intensifican con sus vecinos de Química Analítica y Biología y Producción Vegetal, mientras que se difumina ligeramente con el resto de departamentos vecinos. Al realizar un rastreo de las clases temáticas del JCR a las que pertenecen las revistas utilizadas por éstos (figura 3) podemos comprobar cómo se solapan algunas áreas de conocimiento con otros departamentos, lo que se traduce en una similitud en necesidades de información (revistas científicas) lo que explica la diferencia de tonalidad existente entre las fronteras con los distintos departamentos.

En la figura 4 se ha reflejado la disposición de todas las revistas más usadas en series de 5, y su disposición topológica en los clusters. De esta forma las revistas que aparecen concentradas en los valles corresponden a revistas específicas de un área de conocimiento, o revistas usadas exclusivamente en ese cluster, no necesariamente de gran frecuencia, y revistas con carácter multidisciplinar de mayor frecuencia de uso en ese cluster.

Asimismo se han representado las revistas que constituyen «la colección básica», es decir aquéllas que son las mas citadas, correspondiendo al núcleo más 1 . $^{\mathrm{a}}$ zona de la ley de Bradford, donde efectivamente la disposición topológica de éstas está concentrada en los clusters más productivos y consolidados .

En cuanto a la disposición topológica de las revistas con un F. I. mayor o igual a 6,5 en el núcleo y 1. ${ }^{\mathrm{a}}$ zona, se concentran en los clusters de las Ciencias Biomédicas, correspondiendo en su mayoría a revistas multidisciplinares en este campo del conocimiento (Bioquímica, Farmacología, Microbiología y Fisiología). En lo referente a la disponibilidad en núcleo y primera zona de revistas científicas, los que mayor número de revistas concentran son Ingeniería Química y Energética y Microbiología.

En relación a lo anterior, hallamos una desigual distribución de revistas disponibles, y en cuanto a su localización, vistos los resultados y el nivel de solapamiento 
Figura 3

Esquema de relaciones del cluster correspondiente al departamento de Bioquímica y Biología Molecular

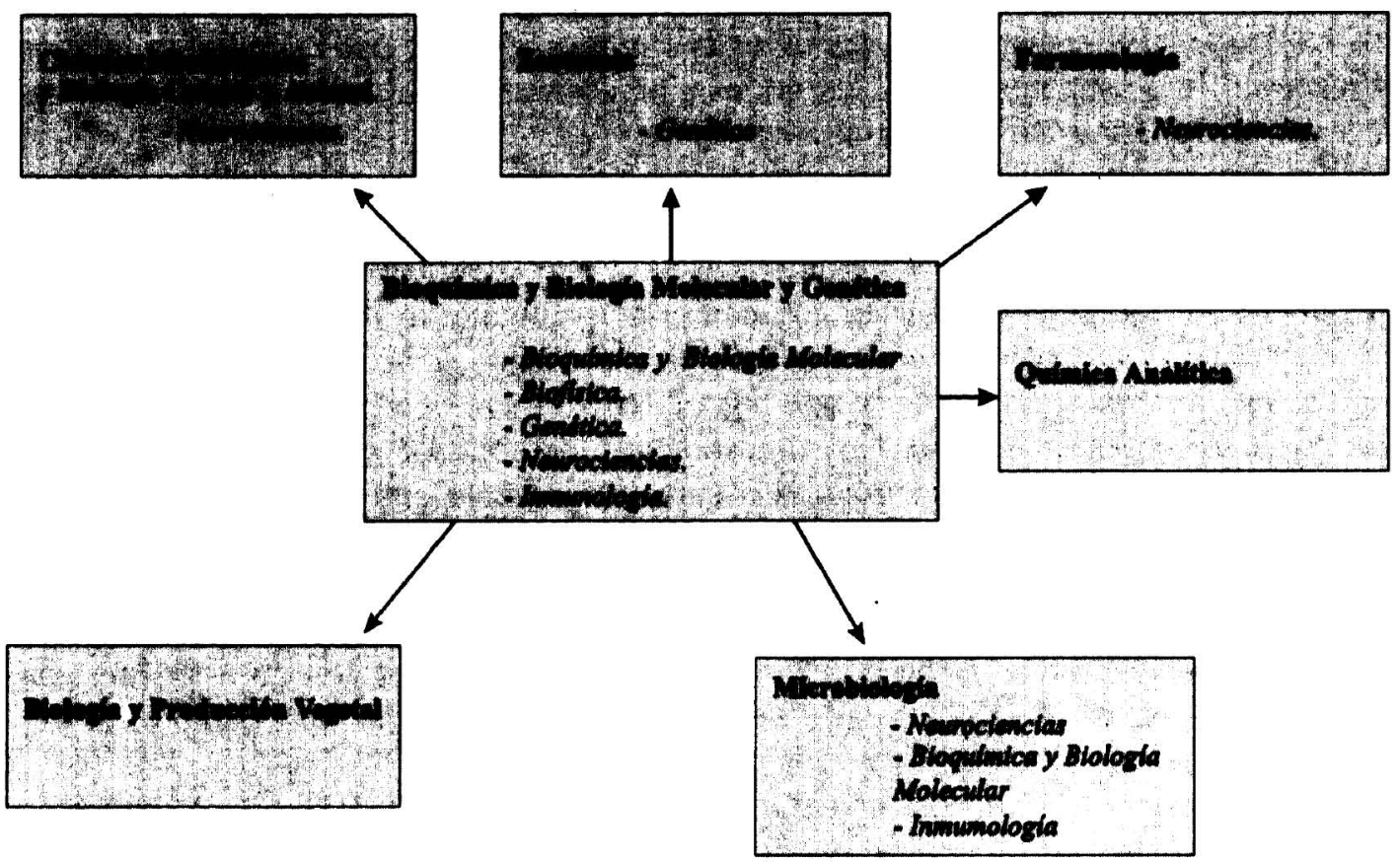

entre áreas de conocimientos, todas las revistas del núcleo y primera zona deberían estar localizadas en una biblioteca que permitiese la accesibilidad general (no olvidemos la estructura departamental, áreas de conocimiento y su presencia en otras titulaciones), recurriendo a ediciones electrónicas que faciliten el acceso a toda la comunidad y donde la ubicación de la edición en papel estaría depositada en la biblioteca (20).

\section{Conclusiones}

Los títulos de revistas utilizados por los científicos presentan una alta dispersión, donde incide la producción de los autores y el índice de colaboración entre éstos, siendo los departamentos más productivos los que más usos hacen de las revistas. La misma dispersión se observa en revistas con usos superiores a diez y el mayor promedio de usos se concentra en las ciencias puras (Física y Química).

En el análisis cualitativo sobre calidad de las revistas utilizadas por los científicos, todos los departamentos superan el factor de impacto promedio de la mediana de las categorías de materias del JCR que son afines a los departamentos, sobresaliendo las áreas biomédicas, que cuadruplican el promedio.

La correlación entre las revistas más usadas por los científicos y el F.I. es inexistente

La disponibilidad que tienen los departamentos de títulos de revistas en bibliotecas de la institución presenta una dispersión, con valores que oscilan del $0 \%$ al $100 \%$.

La colección básica está constituida por 181 revistas (30 en el núcleo y 151 en la primera zona), de modo que el $54,46 \%$ de las citas lo soportan 181 revistas $(7,4 \%$ del total de revistas). 
Figura 4

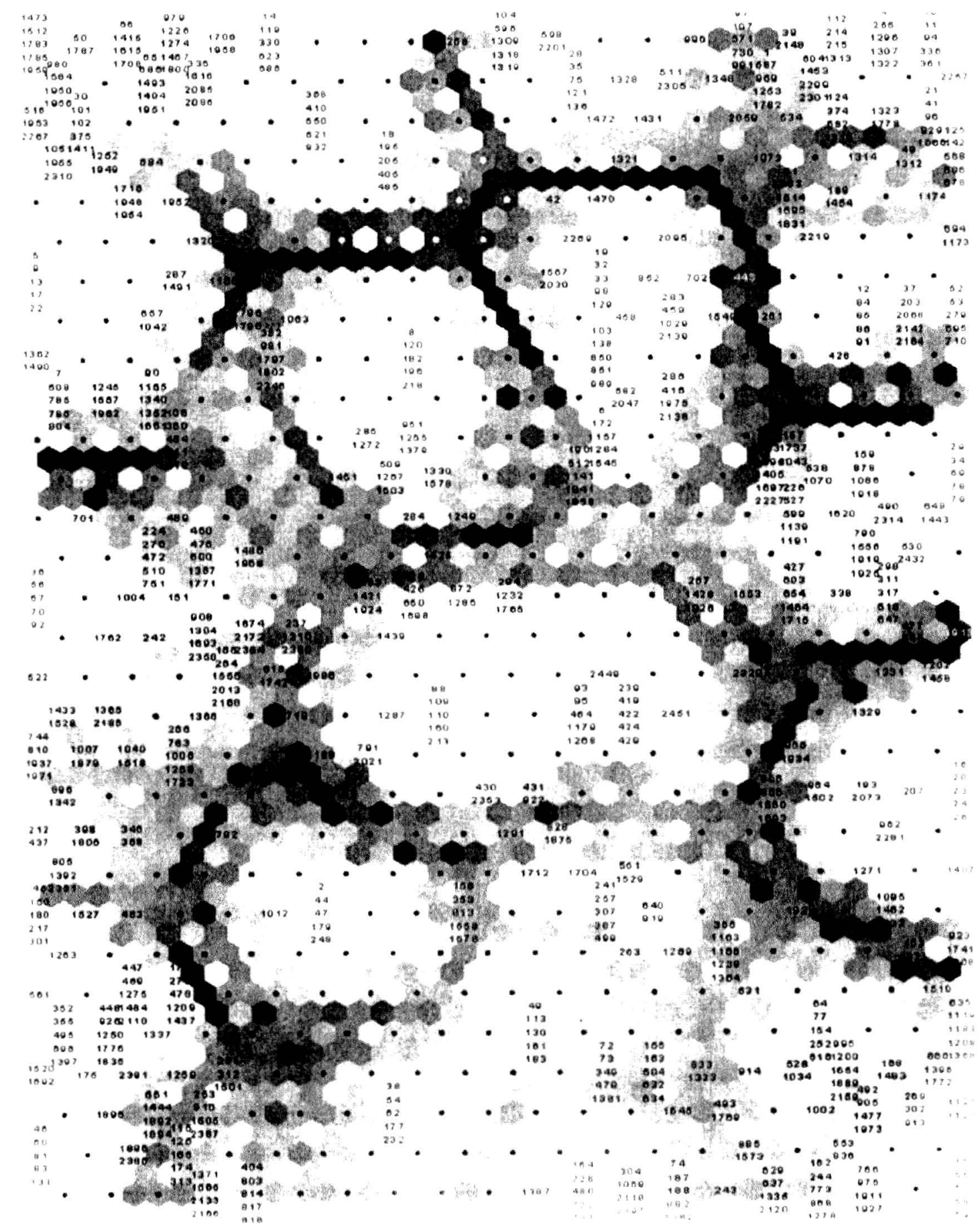

La ley de Bradford favorece los grupos de cientificos mas comsolidades, pero re fleja fielnente el consumo real de informacion.

La correlacion entre citas y usos es muy fuerte con valores superiores al 0,95

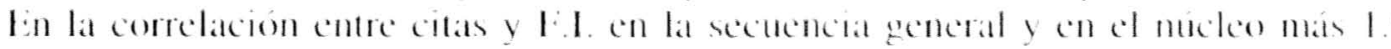
ana se observat una dependencia muy baja. 
Las revistas disponibles en la Institución con más de 10 usos y 5 citas son 90 , que suponen un $33 \%$ y un 3,6\% en relación al total. En el núcleo, el $43 \%$ no está disponible y en la $1 .^{a}$ zona el $75 \%$. En el núcleo más 1 . $^{\mathrm{a}}$ zona sólo está disponible el $30 \%$ del total de revistas que lo conforman.

La cobertura que proporcionaría el núcleo más $1^{\star}$ zona por departamentos, si aceptamos como colección básica de revistas la determinada por éste, sería del $100 \%$ en Fisiología al 3.600\% en Patología y Clínicas Humanas, donde se incluirían casi todas las revistas con más de $\mathbf{1 0}$ usos por departamento, abarcándose también otras con carácter multidisciplinar y otras con usos menores a 10, presentando en general un uso interdepartamental. Estos incrementos se generarían en todos los departamentos menos en Ingenierías Químicas y Energéticas y Fisiología que descenderían por darse la paradoja de tener revistas con uso mayor o igual a 10 pero no mayor o igual a 9 citas (límite de la primera zona).

La disposición espacial de los clusters refleja campos de conocimiento afines en función de los usos de revistas científicas. El compartir áreas de conocimiento (clases temáticas del JCR) se va a traducir en una similitud de necesidades de información.

La disposición topológica de revistas más citadas (colección básica) se concentra en los clusters mas productivos y consolidados (Química Orgánica, Química Física, Ingenierías Químicas y Energéticas y Fisiología).

Las revistas que presentan un mayor factor de impacto en el núcleo más primera zona mayor o igual a 6,5, adoptan una disposición topológica desigual, concentrándose en los clusters de las ciencias biomédicas, correspondiendo a revistas multidisciplinares (Bioquímica, Farmacología, Microbiología y Fisiología).

El uso del algoritmo de Kohonen se adapta a la clasificación del conocimiento a través de revistas científicas, reflejando fielmente los resultados obtenidos de métodos cuantitativos y cualitativos.

Dada la desigual distribución de revistas disponibles se propone como medida prioritaria hacer accesible el núcleo más la primera zona resultante de la aplicación de la ley de Bradford, en donde se concentra el $54 \%$ de la información requerida por los científicos.

Proponer como ubicación óptima la biblioteca institucional y recurrir a ediciones electrónicas de las revistas más usadas por la comunidad científica, dado el nivel de solapamiento interdepartamental y toda vez que los planes de estudio son cada vez mas multidisciplinares.

Los patrones actuales pueden ser buenos predictores del uso futuro; lo que parece razonable debido a la inercia que parece existir en las comunidades científicas, y la tendencia del investigador a encuadrarse dentro de unas líneas de investigación. Por otra parte, los cambios de investigación acaecidos año tras año producen un efecto mínimo en las necesidades y demandas de información.

\section{Bibliografía}

1. BROUDE, J. Journal deselection in an academic environment: a comparison of faculty and librarian choices. Serials Librarian (New York), 1978, v. 3, n. 2, p. 147-166.

2. LANCASTER, F. W. Evaluación de la Biblioteca. Madrid: Anabad, 1996.

3. LANCASTER, F. W. If you want to evaluate your library. London: Library Association, 1988. 
4. HAWTHORN, M. Serials selection and deselection: a survey of North-American academic libraries. Serials Librarian (New York), 1991, v. 21, n. 1, p. 29-45.

5. RASHID, M. F. Bibliometric analysis as a tool in journal evaluation. Serials Librarian (New York), 1991, v. 20, n. 2/3, p.55-64.

6. VALLS PASOLA, A. La evaluación de revistas en una biblioteca universitaria de cara a la cancelación de títulos. Revista Española de Documentación Científica, 1993, v. 16, n. 2, p. 147-156.

7. GONZÁLEZ, W. et al. Experiencia en el desarrollo de una política de selección en un Centro de Información Técnica del sector petrolero. Revista Española de Documentación Cientifica, 1996, v. 19, n. 4, p.427.

8. JIMENEZ-CONTRERAS, E. et al. Determinación de las colecciones básicas de publicaciones periódicas en hemerotecas científicas. Actas de las IV Jornadas Españolas de Documentación Automatizada, Gijón, 1994. Oviedo: Universidad de Oviedo, 1994.

9. ALTUNA-ESTEIBAR, B.; LANCASTER, F. W. Ranking of journal in library and Información Science by research and teaching relatedness. Serials Librarian, 1992, n. 23, p. 110.

10. HAYES, R. M. The distribution of use library materials: analysis of data from de University of Pitsburgh. Library Research, 1981, n. 3, p. 215-260.

11. VÁZQUEZ, M. Perspectivas del empleo de técnicas automatizadas en los estudios bibliométricos. II Encuentro Hispano-Luso de Información Científico-técnica. Salamanca: SEDIC, 1988.

12. KOHONEN, T. Self-organization map. Berlin, Heidelberg: Springer Verlag, 1995.

13. SALTON, G. Mathematics and information retrieval. Journal of Documentation, 35, 1, 129, Marzo 1979.

14. SALTON, G. Automatic information and retrieval. McGraw-Hill, NewYork, 1968.

15. FERREIRO, L. Dispersion de la literatura científica: su ajuste a la ley de Bradford. $R e$ vista Española de Documentación Científica, 1984, 7, p. 89-194.

16. BROOKES, B. C. Bradford's law and the bibliography of science. Nature, 1969, n. 224, p. 953-956.

17. BARRUECO, J. M. Una aproximación a las revistas científicas en formato electrónico. Revista Española de Documentación Cientifica. 1996, v. 19, n. 3, p. 303-313.

18. SCHAFFNER, A. C. The future of scientific journals: lessons from the past. Information Technology and Libraries, 1994, v. 13, n. 4, p.230-247.

19. MARTIN VEGA, A. Las redes neuronales artificiales en la recuperación de la información. Actas de las IV Jornadas Españolas de Documentación Automatizada, Gijón, 1994. Oviedo: Universidad de Oviedo, 1994.

20. REYES-BARRAGÁN, M. J.; GUERRERO BOTE, V.; PULGARÍN GUERRERO, A. Revistas científicas: del formato papel a la edición electrónica en el entorno de la biblioteca universitaria de la UEX. Actas de las 2. Jornadas Andaluzas de Documentación. 1999, noviembre 11-13, Granada, p. 319-333.

\section{Anexo I}

\section{Códigos numéricos asignados a los distintos departamentos}

1. Química Física.

2. Enfermería

3. Bioquímica y Biología Molecular y Genética.

4. Informática.

5. Ciencias Morfológicas y Biología Celular y Animal 
6. Economía Aplicada y Organización Empresarial.

7. Microbiología.

8. Medicina y Sanidad Animal.

9. Zootecnia.

10. Química Orgánica.

11. Física.

12. Fisiología.

13. Ingeniería Química y Energética.

14. Química Inorgánica.

15. Patología y Clínicas Humanas.

16. Farmacología y Psiquiatría.

17. Química Analítica y Electroquímica

18. Biología y Producción Vegetal.

19. Electrónica e Ingeniería Electromecánica.

20. Matemáticas.

21. Especialidades Médico Quirúrgicas. 